\title{
Note on Using the Reciprocal Function for a Linear Inverse Interpolation
}

Let it be required to find the argument, $x$, by linear interpolation from a table of values of $y(x)$. If there is also a table of values of the reciprocal function $\frac{1}{y(x)}$, then a better linear interpolation may result by using the reciprocal function. Frequently, in applications such as in triangle or compound interest problems, $y(x)$ is given as equal to a quotient $\frac{A}{B}$. Hence the solution of $\frac{1}{y(x)}=\frac{B}{A}$ for $x$ by linear interpolation can be made with equal facility. For example, if $\tan x=\frac{A}{B}$ is given for determination of $x$, we might just as easily determine $x$ from the relation $\cot x=\frac{B}{A}$, using the $\cot x$ table. It is the purpose of this note to determine the conditions for which interpolation will be improved by using the reciprocal function.

Suppose that $y(x)$ and $z(x)$ are two monotonic functions such that $y z=1$. Since the slopes of $y$ and $z$ have the relation, $z^{\prime} y+y^{\prime} z=0$, and hence are always opposite in sign, we choose $y$ as the increasing function.

Now the value, $x_{a}$, given by linear interpolation for the argument, $x$, in a table of values of $y(x)$ is given by

$$
x_{a}=x_{1}+\frac{y-y_{1}}{y_{2}-y_{1}}\left(x_{2}-x_{1}\right) \text { where }\left\{\begin{array}{l}
x_{1}<x_{a}<x_{2} \\
y_{1}<y<y_{2} .
\end{array}\right.
$$

(We assume that the function $y(x)$ is positive. Roundoff errors are not considered.) The error, $E_{a}$, due to the interpolation is given by $E_{a}=x_{a}-x$. See [1] for the maximum error inherent in determining a function by linear interpolation from tables. It is given by

$$
\mid \text { error } \mid \leq \frac{\left(x_{2}-x_{1}\right)}{8} M
$$

where $M$ denotes the maximum absolute value of $f^{\prime \prime}(x)$ in the interval $\left(x_{1}, x_{2}\right)$. However, the problem presented in this paper is one of inverse interpolation. Hence this error formula can be applied only after the function is inverted. For compound interest functions, see [2].

The corresponding interpolated value, $x_{b}$, and the error, $E_{b}$, may be determined for the reciprocal function, $z$. (See [3]. It should be noted that this paper is not considering reciprocal differences. We consider only the differences of the reciprocal function.) The condition that the interpolation is improved by using the reciprocal function is given by $\left|E_{b}\right|<\left|E_{a}\right|$. We note that $E_{a}<0(>0)$ if $y^{\prime \prime}>0(<0)$ and $E_{b}>0(<0)$ if $z^{\prime \prime}>0(<0)$ and thus we have the following cases: 
Case I. $y^{\prime \prime}<0$ and $z^{\prime \prime}>0$, then $E_{a}$ and $E_{b}$ are both positive. In this case the result is poorer since always

(1) $E_{b} \equiv x_{1}-x+\frac{z-z_{1}}{z_{2}-z_{1}}\left(x_{2}-x_{1}\right)>x_{1}-x+\frac{y-y_{1}}{y_{2}-y_{1}}\left(x_{2}-x_{1}\right) \equiv E_{a}$.

The difference in the errors is given by

$$
E_{b}-E_{a}=\frac{\left(y-y_{1}\right)\left(y_{2}-y\right)\left(x_{2}-x_{1}\right)}{\left(y_{2}-y_{1}\right) y}
$$

Case II. $y^{\prime \prime}>0$ and $z^{\prime \prime}<0$, then $E_{a}$ and $E_{b}$ are both negative. Inequality (1) remains true, but now $\left|E_{b}\right|<\left|E_{a}\right|$ and hence the interpolation is always improved.

Case III. $y^{\prime \prime}<0$ and $z^{\prime \prime}<0$. This case is impossible. The reason for this is as follows: From $y z=1$, one obtains $z^{\prime} y+y^{\prime} z=0$ and $z^{\prime \prime} y+2 z^{\prime} y^{\prime}+z y^{\prime \prime}=0$. Hence $z^{\prime \prime}+z^{2} y^{\prime \prime}=2\left(z^{\prime}\right)^{2} y$, and since $y>0, y^{\prime \prime}$ and $z^{\prime \prime}$ cannot both be negative. In fact, if $y^{\prime \prime}$ (or $z^{\prime \prime}$ ) is negative, then $z^{\prime \prime}$ (or $y^{\prime \prime}$ ) is positive.

Case IV. $y^{\prime \prime}>0$ and $z^{\prime \prime}>0$, then $E_{a}$ is negative and $E_{b}$ is positive. The inequality is $E_{b}<\left|E_{a}\right|$, giving the condition,

$$
E_{b}-\left|E_{a}\right|=\frac{y-y_{1}}{y_{2}-y_{1}}\left(x_{2}-x_{1}\right)\left(1+\frac{y_{2}}{y}\right)-2\left(x-x_{1}\right)<0
$$

or

$$
E_{b}-\left|E_{a}\right|=\frac{z_{1}-z}{z_{1}-z_{2}}\left(x_{2}-x_{1}\right)\left(1+\frac{z_{2}}{z}\right)-2\left(x-x_{1}\right)<0 .
$$

Some examples follow :

$$
\begin{aligned}
& \text { I }\left\{\begin{array}{lll}
y=\sec x\left(0<x<\frac{\pi}{2}\right) & y^{\prime \prime}>0 & \text { Case II } \\
z=\cos x & z^{\prime \prime}<0 & \text { Result is always improved. }
\end{array}\right. \\
& \text { II }\left\{\begin{array}{lll}
y=\sin x\left(0<x<\frac{\pi}{2}\right) & y^{\prime \prime}<0 & \text { Case I } \\
z=\csc x & z^{\prime \prime}>0 & \text { Result is always poorer. }
\end{array}\right. \\
& \text { III }\left\{\begin{array}{lll}
y=\sqrt{x}(x>0) & y^{\prime \prime}<0 & \text { Case I } \\
z=\left(\frac{1}{\sqrt{x}}\right) & z^{\prime \prime}>0 & \text { Result is always poorer. }
\end{array}\right. \\
& \text { IV }\left\{\begin{array}{lll}
y=e^{x}(x>0) & y^{\prime \prime}>0 & \text { Case IV } \\
z=e^{-x} & z^{\prime \prime}>0 & \text { Result is improved if } \\
\frac{x_{1}+x_{2}}{2}<x<x_{2}
\end{array}\right.
\end{aligned}
$$




$$
\mathrm{V}\left\{\begin{array}{lcl}
y=(1+i)^{n} \text { for } n & y^{\prime \prime}>0 & \text { Case IV } \\
z=(1+i)^{-n}(n>0) & z_{b}-\left|E_{a}\right|=0 \text { if } n=\frac{n_{1}+n_{2}}{2} \\
& & \text { Result is improved if } \\
\frac{n_{1}+n_{2}}{2}<n<n_{2} .
\end{array}\right.
$$

VI $\left\{\begin{array}{l}y=s_{\mathfrak{n}}(i) \text { for } n \\ x=\frac{1}{s_{\mathfrak{n}}}(i) n>0\end{array}\right.$

Case IV

Result is same as for $(1+i)^{n}$.

VII $\left\{\begin{array}{lll}y=a_{\bar{n}}(i) \text { for } n & y^{\prime \prime}<0 & \text { Case I } \\ z=\frac{1}{a_{\bar{n}}}(i)(n>0) & z^{\prime \prime}>0 & \text { Result is always poorer. }\end{array}\right.$

VIII $\begin{cases}y=\tan \theta \text { for }\left(0<\theta<\frac{\pi}{2}\right) & y^{\prime \prime}>0 \quad \text { Case IV } \\ z=\cot \theta & z^{\prime \prime}>0 .\end{cases}$

The error in this example is given by

$$
\left|E_{a}\right|=\left|\theta_{1}+\frac{\tan \theta-\tan \theta_{1}}{\tan \theta_{2}-\tan \theta_{1}}\left(\theta_{2}-\theta_{1}\right)-\theta\right| \text {. }
$$

In this case $\left|E_{a}\right|$ increases as $\theta$ increases from 0 to $\frac{\pi}{2}$ for constant values of $\theta_{2}-\theta_{1}$ and $\theta-\theta_{1}$. That this is true is seen by the relation

$$
\frac{\tan \theta-\tan \theta_{1}}{\tan \theta_{2}-\tan \theta_{1}}=\frac{\sin \left(\theta-\theta_{1}\right) \cos \theta_{2}}{\sin \left(\theta_{2}-\theta_{1}\right) \cos \theta}
$$

Now

$$
\left|E_{a}\left(\theta, \theta_{1}, \theta_{2}\right)\right|=E_{b}\left(\frac{\pi}{2}-\theta, \frac{\pi}{2}-\theta_{1}, \frac{\pi}{2}-\theta_{2}\right)
$$

Hence $E_{b}<\left|E_{a}\right|$ only if $\theta>\frac{\pi}{4}$, and in this case the interpolation is improved; but if $\theta<\frac{\pi}{4}$ the interpolation is poorer.

Michigan State University

East Lansing, Michigan

Hugh E. Stelson.

1. J. B. Scarborough, Numerical Mathematical Analysis, Johns Hopkins Press, 1930, p. 107. 2. Hugh E. STELson, "The accuracy of linear interpolation in tables of the mathematics of finance," $M T A C$, v. 3, 1949, p. 408-412.

3. L. M. Milne-Thomson, The Calculus of Finite Differences, Macmillan and Co., 1933, p. 104-123.

4. W. E. Milne, Numerical Calculus, Princeton Univ. Press, 1949, p. 219-236. 